\title{
MAGNETIC FIELDS IN THE IRREGULAR GALAXY LMC
}

\author{
RICHARD WIELEBINSKI
}

Max-Planck-Institut für Radioastronomie, Auf dem Hügel 69, W-5300 Bonn 1, FRG

November 2, 1992

\begin{abstract}
New radio continuum surveys allowed the determination of the magnetic field in the Large Magellanic Cloud. The magnetic field is filamentary, extending to the south of the 30 Doradus nebula. Some possible explanations for this unusual morphology are discussed.
\end{abstract}

There is very little information on magnetic fields in irregular and dwarf galaxies. The detection of linear polarization in NGC 4449 was reported by Klein and Gräve (1987). A sample of dwarf galaxies was observed by Klein et al. (1991) implying the presence of magnetic fields in all of the studied objects.

The Large Magellanic Cloud is our nearest extragalactic neighbour. In the normal optical plates we see a surprisingly pronounced 'bar'. However, deep plates of the LMC show a very much larger, more symmetric galaxy. One object, the 30 Doradus nebula, (also called the Tarantula nebula), is a very special centre of activity, actually situated at one end of the bar. The classification of the LMC is as an irregular dwarf galaxy.

Optical polarization observations (Schmidt 1970, 1976; Mathewson and Ford 1970) both of stars and photopolarimetric data showed a regular polarization distribution. The initial interpretation of this data led to the postulation of a 'PanMagellanic' magnetic field. A reanalysis of the optical data by Wayte (1990) reinforced the view that the magnetic field threads both the Large and Small Magellanic Cloud.

The studies of magnetic fields in the LMC have experienced a revival since new multi-frequency radio continuum surveys (Haynes et al. 1991) became available. These surveys showed that there are filamentary magnetic fields in the LMC. Furthermore the fields seem to be associated with the 30 Dor nebula and not with the main body of the LMC. This is shown in Figure 1 where the $13 \mathrm{~cm}$ maps from Haynes et al. (1991) are reproduced. In conjunction with data at two additional frequencies Klein et al. (1992) concluded that the two filaments could in fact be part of a loop which goes out of 30 Doradus and returns.

The structure of the LMC has been studied recently by Luks and Rohlfs (1992) in the HI line. They identify two seperate components one which is associated with the main disc of the LMC and the second one which is more filamentary and associated with the 30 Dor nebula. The second component has a lower rotational velocity then the disc component. In addition Luks and Rohlfs argue that the HI component associated with the 30 Doradus must be some $250-400 \mathrm{pc}$ above the disc of the LMC.

The radio continuum data also suggest that the polarized filaments are in front of the main disc LMC. Several HII regions are seen in the direction of the polarized emission but these do not affect the well organised ' $E$ ' vectors. This is only possible if the HII regions are lying behind the highly polarized emission. 

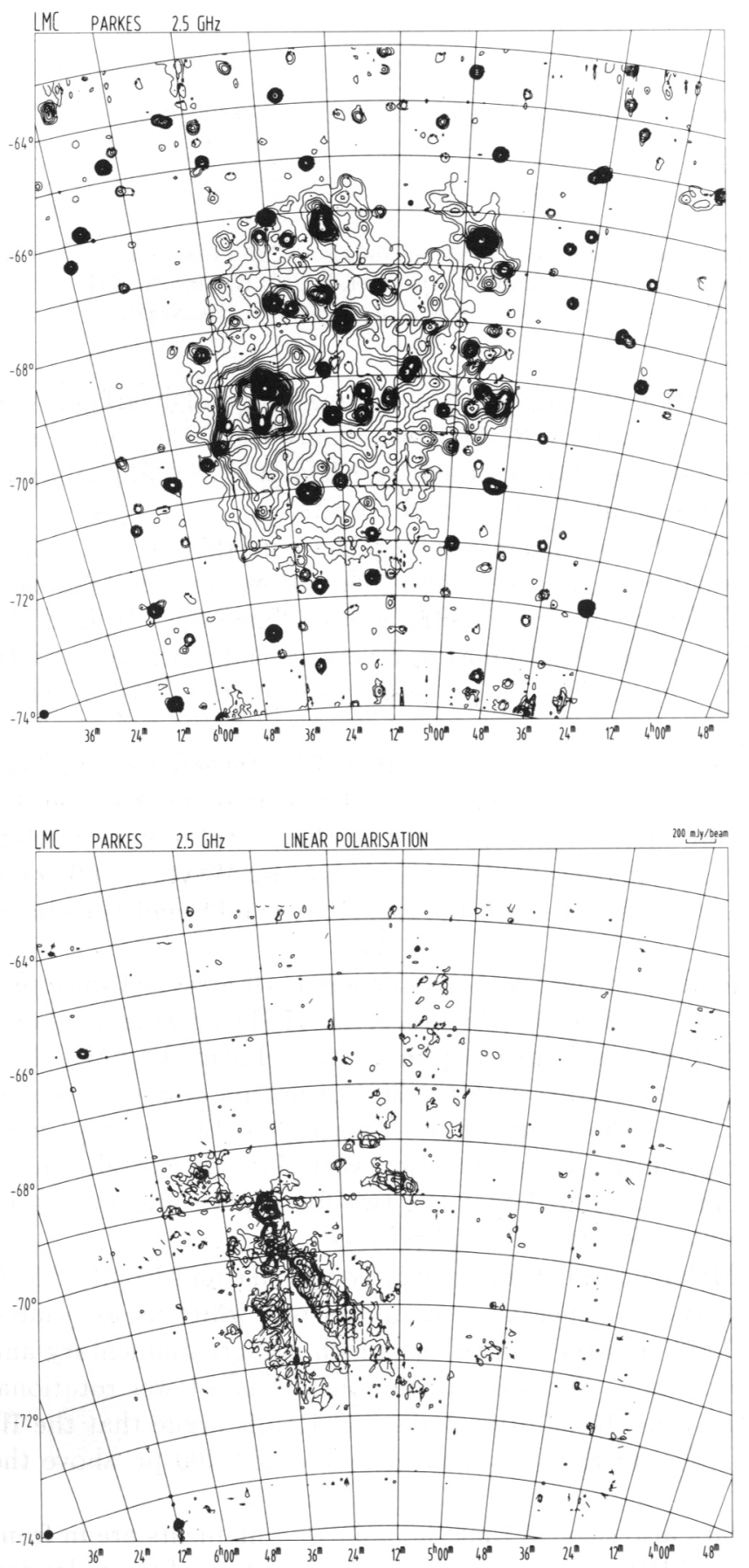

Fig. 1. The total (top) and polarized (bottom) intensity maps at $2.5 \mathrm{GHz}$ from Haynes et al. (1991). 
Fig. 2. A possible morphology of the magnetic field in the LMC.

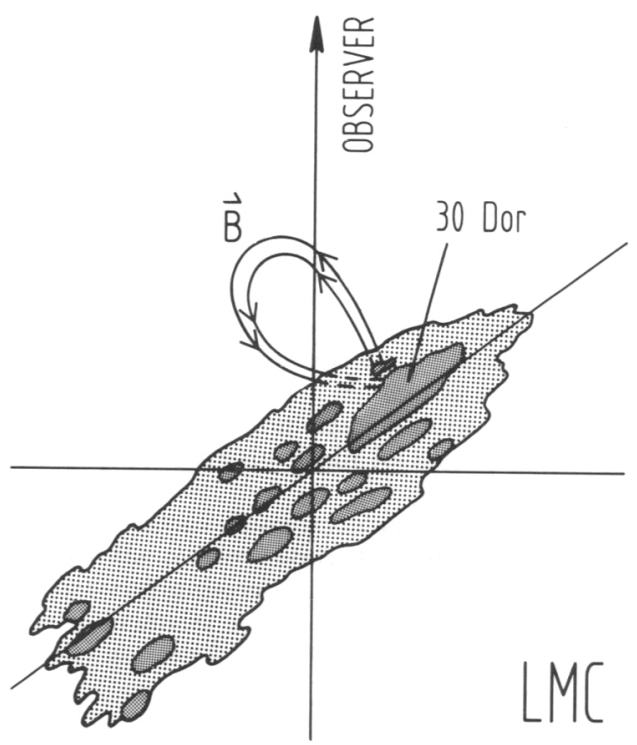

Based on all these data we suggest that the structure of the loop of magnetic field is such that it originates in 30 Doradus nebula and it extends for $\sim 3 \mathrm{kpc}$ towards the main LMC disk. This scenario is shown in Figure 2. We furthermore suggest that this morphology may be a result of the collision between the SMC and the LMC as postulated by Murai and Fujimoto (1980). The passage of the SMC through the disk of the LMC may have led to violent star-forming activity which made the 30 Doradus complex. Furthermore the active region was detached from the main disk of the LMC but did not follow entirely the motion of the SMC and is now $\sim 300$ pc away. A search for a weak compact radio source in 30 Doradus nebula would be helpful in deciding about the reality of the proposed scenario.

\section{References}

Haynes, R.F. et al.: 1991, $A \& A$ 252, 475

Klein, U. and Gräve, R.: 1987, in R. Beck, R. Gräve, ed(s)., Interstellar Magnetic Fields, Springer: Berlin, p. 76

Klein, U., Weiland, H. and Brinks, E.: 1991, $A \& A$ 246, 323

Klein, U., Haynes, R.F., Wielebinski, R., Meinert, D.: 1992, A\&A (submitted)

Luks, T. and Rohlfs, K.: 1992, A\&A 263, 41

Mathewson, D.S. and Ford, V.L.: 1970, $A p J$ 75, 778

Murai, T. and Fujimoto, M.: 1980, PASJ 32, 581

Schmidt, Th.: 1970, $A \& A$ 6, 294

Schmidt, Th.: 1976, A\&AS 24, 357

Wayte, S.R.: 1990, ApJ 355, 473 